\title{
Effect of different doses of aspirin on the prognosis of Kawasaki disease
}

Jinxin Wang ${ }^{1 \dagger}$, Huiqiao Chen ${ }^{1 \dagger}$, Hongying Shi ${ }^{2}$, Xuting Zhang ${ }^{1}$, Yiping Shao ${ }^{1}$, Biyao Hang ${ }^{1}$, Zhipeng Xu' Xing Rong ${ }^{1}$, Maoping $\mathrm{Chu}^{1 *}$ and Huixian Qiu $^{1 *}$

\begin{abstract}
Background: Kawasaki disease (KD) is the leading cause of acquired heart disease in children, and is steadily increasing in prevalence in East Asia. KD is often complicated by coronary artery damage, including dilatation and/ or aneurysms. Aspirin is used with intravenous immunoglobulin (IVIG) to prevent coronary artery abnormalities in $\mathrm{KD}$. However, the role and optimal dose of aspirin remain controversial. Identifying the dose of aspirin in the acute phase will facilitate development of a more appropriate treatment strategy in improving the outcome of KD.
\end{abstract}

Methods: A total of 2369 patients with KD were retrospectively analyzed and divided into three groups according to the aspirin dose: 510 in group 1 (20-29 mg/kg/day), 1487 in group 2 (30-39 mg/kg/day), and 372 in group 3 ( $40-50 \mathrm{mg} / \mathrm{kg} /$ day). The differences in laboratory data, rate of IVIG resistance and coronary artery damage were compared among the groups.

Results: There was no difference in the incidence of coronary artery aneurysms (CAAs) in group 1 compared with groups 2 and 3 (2 weeks of illness: $2.94 \%$ vs. 1.90\% vs. 3.36\%; $3-4$ weeks of illness: $1.94 \%$ vs. $2.32 \%$ vs. 2.65\%). The risk for developing CAA was not reduced at 2 weeks of illness onset in groups 2 and 3 compared with group 1 (adjusted $\mathrm{OR}=1.05,95 \%$ confidence interval: $0.34-3.18$; $\mathrm{aOR}=1.81,95 \% \mathrm{Cl}: 0.42-7.83$ ). Furthermore, the risk for developing CAA was not reduced at 3-4 weeks of illness onset in groups 2 and 3 (aOR=2.63,95\% Cl: 0.61-11.28; $\mathrm{aOR}=0.52,95 \% \mathrm{Cl}: 0.03-9.54)$. There was no significant difference in the rate of IVIG resistance among the groups. Platelet levels after IVIG treatment in group 1 were significantly lower than those in groups 2 and $3\left(522.29 \times 10^{9} / \mathrm{L}\right.$, $544.69 \times 10^{9} / \mathrm{L}$, and $\left.557.77 \times 10^{9} / \mathrm{L}, p=0.013\right)$. C reactive protein of the $30-40 \mathrm{mg} / \mathrm{kg}^{*}$ day group was slightly higher than the other two groups. $(7.76,8.00$, and $7.01 \mathrm{mg} / \mathrm{L}, p=0.028)$.

Conclusions: Aspirin at the dose of $20-29 \mathrm{mg} / \mathrm{kg} /$ day dose not increase the risk of coronary artery damage and IVIG resistance compared with the dose of $30-50 \mathrm{mg} / \mathrm{kg} /$ day. This low dose may have a lower risk for a potential effect on liver function.

Keywords: Aspirin, Kawasaki disease, Dose, Prognosis, Coronary artery damage, Intravenous immunoglobulin

\footnotetext{
*Correspondence: chmping@hotmail.com; qiuhx1053@126.com

† Jinxin Wang and Huiqiao Chen contributed equally to this work.

'Children's Heart Center, The Second Affiliated Hospital and Yuying

Children's Hospital, Institute of Cardiovascular Development and Translational

Medicine, Wenzhou Medical University, Wenzhou 325027, Zhejiang, China

Full list of author information is available at the end of the article
} 


\section{Background}

Kawasaki disease (KD) is a vascular inflammatory disease. KD is currently the most common acquired heart disease, and has already surpassed the rate of rheumatic heart disease [1]. The incidence of KD in Japan and South Korea is the highest in the world, with an incidence of 330 and 194.7 per 100,000 people, respectively. However, the incidence of KD in the United States, the United Kingdom, and Australia ranges from 8.39 to 25 per 100,000 people [2-6]. KD primarily endangers children and can cause coronary artery aneurysms (CAAs). The incidence of CAA has fallen sharply after standardized treatment [7].

The initial treatment of KD with IVIG has generally reached consensus worldwide. However, there is still controversy about the appropriate aspirin dose to be used as a secondary cornerstone treatment. In North America, under the guidance of the America Heart Association (AHA) guidelines, high-dose aspirin $(80-100 \mathrm{mg} / \mathrm{kg} /$ day) is used in the acute phase of KD [7]. In Asian regions under the guidance of the Japanese guidelines, moderate-dose aspirin (30$50 \mathrm{mg} / \mathrm{kg} /$ day) is widely used [8], and the aspirin dose in Western Europe is the same as that in Asia. Some Centers are opting to use low dose aspirin (3$5 \mathrm{mg} / \mathrm{kg} /$ day) [9].

Because of a lack of high-quality, randomized, and controlled trials, each dose has its supporting basis and cannot be completely unified. To balance the risks and benefits of aspirin, we performed a study to determine whether different doses of aspirin affect the outcome of KD including IVIG resistance and coronary artery damage.

\section{Materials and methods \\ Subjects}

We reviewed the data of children who were diagnosed with KD at the Second Affiliated Hospital and Yuying Children's Hospital of Wenzhou Medical University from January 2005 to December 2018. There were initially a total of 2768 children. We included children who were treated with $20-50 \mathrm{mg} / \mathrm{kg} /$ day of aspirin in the study. A total of 42 children who did not use aspirin and 301 children who received less than $20 \mathrm{mg} / \mathrm{kg} /$ day or more than $50 \mathrm{mg} / \mathrm{kg} /$ day were excluded. At the same time, we excluded seven patients without cardiac ultrasound results and 49 in whom IVIG use was unknown. Finally, 2369 children were included in this study. The study flowchart is shown supplementary Fig. 1. All children were divided into the following groups according to the aspirin dose: group 1 (20-29 mg/kg/day), group 2 (30-39 mg/kg/ day), and group 3 (40-50 $\mathrm{mg} / \mathrm{kg} /$ day).

\section{Data collection}

We collected the following data from the electronic medical record: weight, age (months), gender, time of IVIG treatment (the number of days that patients treated with IVIG after the onset of disease), and the regime of IVIG treatment. Laboratory data before and after IVIG treatment were recorded, including the white blood cell count (WBC), absolute neutrophil count (ANC), levels of hemoglobin $(\mathrm{Hb})$, alanine aminotransferase (ALT), aspartate aminotransferase (AST), C-reactive protein (CRP), albumin, fibrinogen (FIB), and D-dimer, and the erythrocyte sedimentation rate (ESR). Echocardiogram examinations were performed at 2 and 3-4 weeks after onset of illness.

\section{Criteria for diagnosis of KD}

The diagnosis of KD was based on the 2017 AHA guidelines [7] as follows: (1) fever $\geq 5$ days, (2) eye conjunctival hyperemia, (3) oral changes, (4) polymorphic rash, (5) hand and foot sclerosis or peeling, and (6) cervical lymphadenopathy.

\section{Resistance to IVIG}

IVIG resistance was defined as when body temperature did not fall to normal $48 \mathrm{~h}$ after the end of IVIG infusion, or increased again above $38.0^{\circ} \mathrm{C}$ [10].

\section{Coronary artery damage}

CAA was defined as a coronary artery diameter $\geq 4$ $\mathrm{mm}[11]$.

\section{Statistical analysis}

Statistical analysis were performed by IBM SPSS (version 23). Continuous variables are shown by the mean \pm standard deviation or median and interquartile range. Categorical variables are expressed by numbers and percentages. The chi-square test was used to compare categorical variables. The associations between aspirin doses and coronary artery damage or IVIG resistance were analyzed using multiple logistic regression. A $p$ value of $<0.05$ was considered statistically significant.

\section{Results}

Demographic characteristics and IVIG treatment options Of 2369 children diagnosed with KD in the study, 510 received $20-29 \mathrm{mg} / \mathrm{kg} /$ day of aspirin treatment, 1487 received $30-39 \mathrm{mg} / \mathrm{kg} /$ day, and 372 received $40-50 \mathrm{mg} /$ $\mathrm{kg} /$ day. There were significant differences in mean weight and age between the three groups (both $p<$ 0.001). There were also significant differences in the proportion of no use of IVIG among the three groups, among which group 1 had the highest proportion, followed by group 2 and then group 3 (5.29\% vs 3.09, 
$1.34 \%, p<0.001)$. No significant difference was found with respect to treatment time of IVIG and gender among the three groups (Table 1).

\section{Laboratory tests before IVIG therapy}

$\mathrm{Hb}$ levels in group 1 were significantly higher than those in the other two groups $(p<0.001$, Table 2$)$. There were no significant differences in the values of the WBC, PLT, ANC, CRP, and the ESR. A liver function test showed that albumin levels were highest in group 1 and lowest in group $3(\mathrm{p}<0.001)$. ALT and AST levels were not significantly different among the three groups. For coagulation measurements, except for FIB levels, which were significantly different among the three groups $(p=0.002)$, the Prothrombin time (PT), Activated partial thromboplastin time (APTT), Thrombin time (TT), and D-dimer levels were not significantly different among the three groups.

\section{Laboratory tests after IVIG therapy}

Patients with $\mathrm{KD}$ had higher $\mathrm{Hb}$ levels in group 1, followed by group 2 and then group $3(p<0.001$, Table 3). PLT levels in group 1 were significantly lower than those in groups 2 and $3(p=0.013)$. CRP levels were significantly different among the three groups $(p=$ 0.028). Albumin levels were significantly higher in group 1 compared with the other two groups $(p<0.001)$. Similar to albumin levels, FIB levels in group 1 were higher compared with those in the other two groups $(\mathrm{p}<$ 0.001). However, ALT, AST, and D-dimer levels, and the $\mathrm{PT}, \mathrm{APTT}$, and TT were not significantly different among the three groups.

\section{CAA and IVIG resistance}

The proportion of IVIG resistance was not significantly different among the three groups (Table 4). Additionally, the incidence of CAA was not different among the three groups at 2 weeks and 3-4 weeks after onset of illness.

\section{Effect of aspirin on CAA and IVIG resistance}

Multiple logistic regression was used to analyze the relationship between the dose of aspirin and the risk of CAA or IVIG resistance after adjusting for age, weight, sex, IVIG treatment options, IVIG treatment time, $\mathrm{Hb}$ levels, albumin levels, and FIB levels (Table 5). Intriguingly, there was no significant correlation between the dose of aspirin and the prevalence of CAA at 2 weeks or 3-4 weeks after onset of illness, even after adjusting for the factors of age, weight, sex, IVIG treatment options, IVIG treatment time, Hb levels, albumin levels, and FIB levels before aspirin treatment. Two weeks after the onset of illness, treatment with $30-339 \mathrm{mg} / \mathrm{kg} /$ day of aspirin was associated with an odds ratio of 1.05 for CAA (95\% confidence interval [CI]: 0.34-33.18, $p=0.9352$ ), whereas $40-350 \mathrm{mg} / \mathrm{kg} /$ day was associated with an odds ratio of 1.81 (95\% CI: $0.42-37.83, p=0.4273$ ). The risk difference of CAA at 3-34 weeks after onset of illness adjusted for potential confounders was 2.63 (95\% CI: $0.61-11.28, p=0.1921)$ in group 2 and 0.52 (95\% CI: $0.03-9.54, p=0.6589$ ) in group 3 . Analysis that was nonadjusted or adjusted for the same related factors also showed no correlation between the risk of IVIG resistance and doses of aspirin combination therapy.

\section{Discussion}

Application of aspirin in KD was reported earlier than that of IVIG [12]. Previous studies [13] have suggested that high-dose aspirin combined with IVIG is beneficial

Table 1 Demographic characteristics

\begin{tabular}{|c|c|c|c|c|}
\hline Group & 1 & 2 & 3 & $P$-value \\
\hline $\bar{N}$ & 510 & 1487 & 372 & \\
\hline Weight (kg) & $11.50(10.50-15.45)$ & $11.30(9.00-14.00)$ & $10.50(7.50-13.00)$ & $<0.001$ \\
\hline Age (month) & $22.14(14.83-39.93)$ & $19.43(10.66-35.14)$ & 15.79 (6.45-29.30) & $<0.001$ \\
\hline IVIG treat time (day) & $6.00(6.00-8.00)$ & $6.00(6.00-7.00)$ & $6.00(6.00-7.00)$ & 0.574 \\
\hline SEX & & & & 0.574 \\
\hline Male & $337(66.08 \%)$ & 948 (63.75\%) & 244 (65.59\%) & \\
\hline Female & $173(33.92 \%)$ & 539 (36.25\%) & $128(34.41 \%)$ & \\
\hline IVIG treat option & & & & 0.004 \\
\hline No use & 27 (5.29\%) & 46 (3.09\%) & $5(1.34 \%)$ & \\
\hline $1 \mathrm{~g}$ or $2 \mathrm{~g}$ options & 483 (94.71\%) & 1441 (96.91\%) & 367 (98.66\%) & \\
\hline
\end{tabular}

Data are shown as number (N), mean (SD), or median (Q1-Q3)/N (\%)

IVIG: intravenous immunoglobulin

Group 1: patients with Kawasaki disease treated with aspirin at $20-30 \mathrm{mg} / \mathrm{kg} / \mathrm{d}$ in the acute stage

Group 2: patients with Kawasaki disease treated with aspirin at $30-40 \mathrm{mg} / \mathrm{kg} / \mathrm{d}$ in the acute stage

Group 3: patients with Kawasaki disease treated with aspirin at $40-50 \mathrm{mg} / \mathrm{kg} / \mathrm{d}$ in the acute stage 
Table 2 Laboratory tests before aspirin treatment

\begin{tabular}{|c|c|c|c|c|}
\hline Group & 1 & 2 & 3 & $P$-value \\
\hline $\mathrm{N}$ & 510 & 1487 & 372 & \\
\hline WBC1 $(10 \wedge 9 / L)$ & 15.15 (11.91-19.37) & 15.57 (12.24-19.80) & $15.42(12.70-19.86)$ & 0.276 \\
\hline $\mathrm{HB1}(\mathrm{g} / \mathrm{L})$ & $111.64 \pm 11.34$ & $110.42 \pm 11.34$ & $108.48 \pm 11.05$ & $<0.001$ \\
\hline $\operatorname{PLT1}(10 \wedge 9 / L)$ & $379.24 \pm 135.27$ & $388.75 \pm 136.16$ & $388.44 \pm 143.93$ & 0.392 \\
\hline ANC1 $(10 \wedge 9 / L)$ & $10.79 \pm 5.15$ & $10.76 \pm 4.99$ & $10.64 \pm 4.80$ & 0.903 \\
\hline CRP1 (mg/L) & $70.10(35.00-113.87)$ & $75.00(42.49-118.01)$ & $81.40(47.55-117.00)$ & 0.196 \\
\hline $\operatorname{ESR} 1(\mathrm{~mm} / \mathrm{h})$ & $37.50 \pm 17.14$ & $36.99 \pm 16.90$ & $35.84 \pm 17.56$ & 0.412 \\
\hline $\operatorname{ALT1}(\mathrm{u} / \mathrm{L})$ & $35.00(17.00-91.00)$ & 34.00 (18.00-92.50) & $33.00(19.00-75.00)$ & 0.552 \\
\hline $\operatorname{AST1}(\mathrm{u} / \mathrm{L})$ & $33.50(25.00-50.25)$ & $31.50(24.00-51.00)$ & $29.00(24.00-52.00)$ & 0.225 \\
\hline Albumin 1(g/L) & $36.52 \pm 6.26$ & $36.19 \pm 6.28$ & $34.63 \pm 6.58$ & $<0.001$ \\
\hline PT1(s) & $13.82 \pm 0.99$ & $13.82 \pm 1.05$ & $13.97 \pm 1.63$ & 0.165 \\
\hline APTT1(s) & $43.34 \pm 5.94$ & $43.71 \pm 6.71$ & $43.11 \pm 5.99$ & 0.331 \\
\hline $\mathrm{FIB1}(\mathrm{g} / \mathrm{L})$ & $1.23(0.85-2.16)$ & $1.43(0.87-2.16)$ & $1.37(0.82-2.28)$ & 0.002 \\
\hline$\Pi 1(\mathrm{~s})$ & $14.84 \pm 0.90$ & $14.89 \pm 1.23$ & $14.87 \pm 1.09$ & 0.804 \\
\hline D-Dimers1 (ug/ml) & $1.23(0.85-2.16)$ & $1.43(0.87-2.16)$ & $1.37(0.82-2.28)$ & 0.328 \\
\hline
\end{tabular}

Continuous data are presented as mean \pm SD if data are normally distributed and expressed as median (interquartile range) if data are not normally distributed WBC white blood cell count, ANC absolute neutrophil count, CRP C reactive protein, ESR erythrocyte sedimentation rate, ALT alanine aminotransferase, AST aspartate aminotransferase, PT prothrombin time, APTT activated partial thromboplastin time, FIB fibrinogen, $\pi$ thrombin time, $H b$ hemoglobin, $P L T$ platelet Count

for preventing coronary damage. Subsequent studies have indicated that coronary damage is only negatively correlated with the dose of IVIG, but not associated with aspirin [14]. However, to date, Japanese [15] and American [7] guidelines still recommend the use of moderatedose aspirin $(30-0 \mathrm{mg} / \mathrm{kg} /$ day $)$ and high-dose aspirin $(80-100 \mathrm{mg} / \mathrm{kg} /$ day), respectively. Clinically, because of the pharmaceutical dosage form, tablet aspirin is usually
$50 \mathrm{mg}, 100 \mathrm{mg}$, but the weight of the children varies. Clinical use of guidelines recommend $30-50 \mathrm{mg} / \mathrm{kg}$ of body weight, but the actual accurate calculation may be 20-50 mg. Coupled with the actual practice habits of different centers, some centers rapidly reduce the dose of aspirin to $3-5 \mathrm{mg} / \mathrm{kg} /$ day after being afebrile, and even some patients with atypical KD without fever before IVIG use usually have a low dose $(3-5 \mathrm{mg} / \mathrm{kg} /$ day $)$.

Table $\mathbf{3}$ Laboratory tests after aspirin treatment

\begin{tabular}{|c|c|c|c|c|}
\hline Group & 1 & 2 & 3 & $P$-value \\
\hline $\mathrm{N}$ & 510 & 1487 & 372 & \\
\hline WBC2(10^9/L) & $8.37 \pm 3.39$ & $8.67 \pm 3.36$ & $8.94 \pm 3.90$ & 0.065 \\
\hline $\mathrm{HB} 2(\mathrm{~g} / \mathrm{L})$ & $109.80 \pm 11.34$ & $108.46 \pm 10.55$ & $106.32 \pm 10.71$ & $<0.001$ \\
\hline PLT2(10^9/L) & $522.29 \pm 173.58$ & $544.69 \pm 172.46$ & $557.77 \pm 193.50$ & 0.013 \\
\hline ANC2(10^9/L) & $2.44(1.68-3.93)$ & $2.60(1.66-3.93)$ & $2.45(1.56-3.76)$ & 0.416 \\
\hline $\mathrm{CRP} 2(\mathrm{mg} / \mathrm{L})$ & $7.76(3.00-14.42)$ & $8.00(3.83-15.18)$ & 7.01 (3.00-14.28) & 0.028 \\
\hline $\operatorname{ESR2}(\mathrm{mm} / \mathrm{h})$ & $35.57 \pm 19.49$ & $35.82 \pm 19.64$ & $32.48 \pm 16.86$ & 0.439 \\
\hline $\operatorname{ALT2}(\mathrm{u} / \mathrm{L})$ & $27.00(18.00-43.00)$ & $28.00(19.00-44.00)$ & $27.00(18.00-46.75)$ & 0.606 \\
\hline AST2(u/L) & $42.00(35.00-55.00)$ & $42.00(35.00-55.00)$ & $43.00(35.00-61.00)$ & 0.456 \\
\hline Albumin2(g/L) & $36.01 \pm 4.30$ & $35.40 \pm 4.74$ & $34.40 \pm 4.55$ & $<0.001$ \\
\hline PT2(s) & $12.82 \pm 0.91$ & $12.81 \pm 0.78$ & $12.81 \pm 0.69$ & 0.978 \\
\hline APTT2(s) & $39.51 \pm 6.13$ & $39.69 \pm 5.67$ & $38.92 \pm 5.26$ & 0.251 \\
\hline $\mathrm{FIB} 2(\mathrm{~g} / \mathrm{L})$ & $4.19 \pm 0.92$ & $4.09 \pm 0.94$ & $3.86 \pm 1.00$ & $<0.001$ \\
\hline$\pi 2(s)$ & $17.38 \pm 1.41$ & $17.41 \pm 1.17$ & $17.34 \pm 1.24$ & 0.780 \\
\hline D-Dimers2 (ug/ml) & $0.98(0.71-1.49)$ & $0.92(0.68-1.42)$ & $0.88(0.62-1.47)$ & 0.198 \\
\hline
\end{tabular}

Continuous data are presented as mean \pm SD if data are normally distributed and expressed as median (interquartile range) if data are not normally distributed WBC white blood cell count, $A N C$ absolute neutrophil count, CRP C reactive protein, ESR erythrocyte sedimentation rate, $A L T$ alanine aminotransferase, $A S T$ aspartate aminotransferase, $P T$ prothrombin time, $A P T T$ activated partial thromboplastin time, FIB fibrinogen, $T T$ thrombin time, $H b$ hemoglobin, $P L T$ platelet Count 
Table 4 Outcome

\begin{tabular}{lllll}
\hline Group & 1 & 2 & 3 & $P$-value \\
\hline IVIG-unresponse & $118(24.48 \%)$ & $344(23.89 \%)$ & $98(26.70 \%)$ & 0.534 \\
CAA(2 weeks) & $14(2.94 \%)$ & $27(1.90 \%)$ & $12(3.36 \%)$ & 0.147 \\
CAA(3-4 weeks) & $7(1.94 \%)$ & $26(2.32 \%)$ & $7(2.65 \%)$ & 0.843
\end{tabular}

CAA coronary artery aneurysm, IVIG intravenous immunoglobulin

Therefore, we took into account the case of a dose $>20$ $\mathrm{mg} / \mathrm{kg} /$ day in the acute phase of KD. In the present study, no significant difference was found in gender and IVIG treatment time among the groups. The difference found in age is related to the true clinical situation in the case of low-weight children and aspirin dosage forms. With a younger age and lower weight, the relative value of aspirin/weight is greater. At the same time, in younger children, clinicians are more careful and the rate of IVIG use is higher.

In recent years, many studies have been performed to determine the optimum dose of aspirin, and they have shown that the current recommended dose is not reasonable. Ogata et al. reported that there was no significant difference between moderate and high aspirin dose in KD [16]. A multicenter, randomized, single blind trial in Taiwan also showed that there was no significant difference in short-term coronary lesions of KD between high-dose and low-dose aspirin in the acute phase [17]. Huang et al. [18] reported that low-dose aspirin and moderate-dose aspirin had no effect on coronary artery damage in $\mathrm{KD}$, although they did not show cardiac Bultrasound time. Recent reports by Brooks et al. And Zheng et al. demonstrate that low-dose aspirin has there was no no significant difference in fever days and coronary artery damage rate between low-dose and traditional

Table 5 Multiple regression analyses

\begin{tabular}{lll}
\hline \multicolumn{2}{c}{ Non-adjusted } & Adjusted \\
\hline IVIG-unresponse & & 1.0 \\
Group 1 & 1.0 & $1.07(0.77,1.49) 0.6958$ \\
Group 2 & $0.97(0.76,1.23) 0.7922$ & $1.17(0.75,1.85) 0.4895$ \\
Group 3 & $1.12(0.82,1.53) 0.4617$ & \\
CAA(2 weeks) & & 1.0 \\
Group 1 & 1.0 & $1.05(0.34,3.18) 0.9352$ \\
Group 2 & $0.64(0.33,1.23) 0.1840$ & $1.81(0.42,7.83) 0.4273$ \\
Group 3 & $1.15(0.53,2.52) 0.7261$ & \\
CAA(3-4 weeks) & & 1.0 \\
Group 1 & 1.0 & $2.63(0.61,11.28) 0.1921$ \\
Group 2 & $1.20(0.51,2.78) 0.6785$ & $0.52(0.03,9.54) 0.6589$ \\
Group 3 & $1.37(0.48,3.96) 0.5572$ &
\end{tabular}

Data are shown as $\mathrm{OR}(95 \% \mathrm{Cl}) \mathrm{p}$ value

CAA Coronary artery aneurysm, IVIG intravenous immunoglobulin The model was adjusted for age, weight, sex; IVIG treatment options, IVIG treatment time, $\mathrm{Hb}$ levels, albumin levels, and FIB levels high-dose aspirin dose groups $[19,20]$. This finding suggested that increasing the aspirin dose in the acute phase of KD may not needed. Furthermore, Migally et al. [21] found that the duration of high-dose aspirin had no relationship with long-term prognosis of the coronary artery. This evidence suggests that high-, moderate-, and low-dose aspirin appear to have no significant difference in the prognosis of $\mathrm{KD}$. Pi et al. [22] reported that 11dehydrothromboxane B2, the final product of the thromboxaneA2 pathway, was not reduced by using aspirin. Additionally, conflicting conclusions were reached by clinical researchers who showed that administration of different doses of aspirin was associated with protection against CAA. Dhanrajani et al. [23] found that IVIG resistance easily occurs with low-dose aspirin. Kim et al. [24] reported that moderate-high-dose aspirin was a risk factor for coronary artery CAA after adjusting for various factors compared with low-dose aspirin. In the present study, our findings showed that there was no significant differences in the incidence of CAA and the proportion of IVIG resistance between KD children who received different doses of aspirin. Our logistic analysis indicated that after adjusting for age, weight, sex, IVIG treatment options, IVIG treatment time, Hb levels, albumin levels, and FIB levels, the risk of CAA was not different among the three groups, indicating that there was no significant correlation between the dose of aspirin and the prevalence of CAA.

As a classic non-steroidal anti-inflammatory drug with acetylsalicylic acid, clinically, aspirin is widely used in preventing cardiovascular disease and postoperative thrombosis. Remarkably, aspirin combines anti-inflammatory (high dose) effects and anti-platelet (low dose) effects. Aspirin inhibits prostaglandin synthetase, reducing prostaglandin production to achieve anti-inflammatory and analgesic effects [25]. The antiinflammatory effect of aspirin increases with dose accumulation. CRP levels proportionally reflect the grade of inflammation. In the present study, CRP levels were comparable among the groups. But the CRP levels of Group $3(40-50 \mathrm{mg} / \mathrm{kg} /$ day $)$ were lower than those of the other two groups. This finding indicates that relatively high doses of aspirin have better anti-inflammatory effects, although after treatment, CRP levels in the three groups all fell to within the normal range. Aspirin inhibits the Cyclooxygenase pathway and disrupts production of thromboxane thromboxaneA2, thereby reducing platelet aggregation and formation of a thrombus [26]. In the present study, significant differences were also found in PLT levels among three goups, the PLT count after treatment of $20-29 \mathrm{mg} / \mathrm{kg} /$ day of aspirin was the lowest among the groups, and to some extent, the relatively low dose of aspirin achieved an antiplatelet effect. 
Additionally, in the human body, aspirin is mainly absorbed in the stomach and small intestine, and rapidly hydrolyzed to salicylic acid. The rate of aspirin binding to plasma albumin is approximately $80-90 \%$. However, during the acute phase of $\mathrm{KD}$, the inflammatory responses of the gastrointestinal tract and a decrease in serum albumin levels lead to decreased efficacy of aspirin. After inflammation is relieved, gastrointestinal function and albumin levels are rapidly restored, which may even lead to drug toxicity [27]. Our results showed that $\mathrm{Hb}$ and albumin levels were significantly higher, and FIB levels were significantly lower in group 1 than those in the other two groups before treatment, while $\mathrm{Hb}$ and albumin levels in the three groups after treatment were the same as those before treatment. This finding suggested that the aspirin dose had little effect on these variables. While equally effective in therapeutic benefits, lowdose aspirin produces fewer side effects compared with traditional relatively high-dose aspirin.

Limitations of this study include its single-center and retrospective nature that is prone to indication bias, as well as a loss of follow-up data. However, our study reflects the real clinical condition. Additionally, the $\mathrm{Z}$ value could not be used to calibrate coronary damage results because of a lack of height data of children with $\mathrm{KD}$. Therefore, a larger and more in-depth study is required and $\mathrm{Z}$ values should be used as possible variations. To the best of our knowledge, no prospective study has been performed to minimize moderate-dose aspirin for preventing CAA in KD.

In conclusion, our data show that in clinical practice, the commonly recommended dosage of $30-50 \mathrm{mg} / \mathrm{kg} /$ day of aspirin is not better than $20-29 \mathrm{mg} / \mathrm{kg} /$ day.

\section{Supplementary information}

Supplementary information accompanies this paper at https://doi.org/10. 1186/s12969-020-00432-x.

Additional file 1: Figure 1. Study flow diagram.

\section{Authors' contributions}

All authors made substantial contributions to the conception or design of the work, data acquisition, analysis or interpretation of data. All authors read and approve the final manuscript.

Availability of data and materials

The datasets used and/or analysed during the current study are available from the corresponding author on reasonable request.

\section{Ethics approval and consent to participate}

The study was approved by the local ethics committee, The Second Affiliated Hospital and Yuying Children's Hospital, China.

\section{Consent for publication}

Not applicable.

\section{Competing interests}

The authors declare that they have no competing interests.

\section{Author details}

${ }^{1}$ Children's Heart Center, The Second Affiliated Hospital and Yuying Children's Hospital, Institute of Cardiovascular Development and Translational Medicine, Wenzhou Medical University, Wenzhou 325027, Zhejiang, China. ${ }^{2}$ Department of Preventive Medicine, School of Enviromental Science and Public Health, WenZhou Medical University, Wenzhou 325035, Zhejiang Province, People's Republic of China.

Received: 6 November 2019 Accepted: 6 May 2020

Published online: 11 June 2020

\section{References}

1. Burns JC, Glode MP. Kawasaki syndrome. Lancet. 2004;364:533-44

2. Kim GB, Park S, Eun LY, et al. Epidemiology and clinical features of Kawasaki disease in South Korea, 2012-2014. Pediatr Infect Dis J. 2017;36:482-5.

3. Saundankar J, Yim D, Itotoh B, et al. The epidemiology and clinical features of Kawasaki disease in Australia. Pediatrics. 2014;133:E1009-14.

4. Harnden A, Mayon-White R, Perera R, et al. Kawasaki disease in England ethnicity, deprivation, and respiratory pathogens. Pediatr Infect Dis J. 2009;28:21-4.

5. Chang RKR. The incidence of Kawasaki disease in the United States did not increase between 1988 and 1997. Pediatrics. 2003;111:1124-5.

6. Makino N, Nakamura Y, Yashiro M et al. The nationwide epidemiologic survey of Kawasaki disease in Japan, 2015-2016. Pediatr Int 2019.

7. McCrindle BW, Rowley AH, Newburger JW, et al. Diagnosis, treatment, and Long-term Management of Kawasaki Disease: a scientific statement for health professionals from the American Heart Association. Circulation. 2017; 135:e927-99.

8. Group JCSJW. Guidelines for diagnosis and management of cardiovascular sequelae in Kawasaki disease (JCS 2013). Digest version Circ J. 2014;78:2521-62.

9. Dallaire F, Fortier-Morissette Z, Blais S, et al. Aspirin dose and prevention of coronary abnormalities in Kawasaki disease. Pediatrics. 2017;139.

10. Fu PP, Du ZD, Pan YS. Novel predictors of intravenous immunoglobulin resistance in Chinese children with Kawasaki disease. Pediatr Infect Dis J. 2013;32:e319-23.

11. Fu S, Gong F, Xie C, et al. S100A12 on circulating endothelial cells surface in children with Kawasaki disease. Pediatr Res. 2010;68:165-8.

12. Kato H, Koike S, Yokoyama T. Kawasaki disease: effect of treatment on coronary artery involvement. Pediatrics. 1979;63:175-9.

13. Newburger JW, Takahashi M, Beiser AS, et al. A single intravenous infusion of gamma globulin as compared with four infusions in the treatment of acute Kawasaki syndrome. N Engl J Med. 1991;324:1633-9.

14. Terai M, Shulman ST. Prevalence of coronary artery abnormalities in Kawasaki disease is highly dependent on gamma globulin dose but independent of salicylate dose. J Pediatr. 1997;131:888-93.

15. Research Committee of the Japanese Society of Pediatric C, Cardiac Surgery Committee for Development of Guidelines for Medical Treatment of Acute Kawasaki D. Guidelines for medical treatment of acute Kawasaki disease: report of the research Committee of the Japanese Society of pediatric cardiology and cardiac surgery (2012 revised version). Pediatr Int. 2014;56:135-58.

16. Ogata S, Tremoulet AH, Sato Y, et al. Coronary artery outcomes among children with Kawasaki disease in the United States and Japan. Int J Cardiol. 2013;168:3825-8.

\footnotetext{
Acknowledgements

The work was supported by National Natural Science Foundation for young scientists (81502893), the Natural Science Foundation of China (No. $81970435,81770502)$ and Zhejiang Provincial Science and Technology Project of Traditional Chinese Medicine (No. 2018ZZ019), Zhejiang Province Science and Technology Agency (LY19H260004,2014C33160).
} 
17. Kuo HC, Guo MMH, Lo MH, et al. Effectiveness of intravenous immunoglobulin alone and intravenous immunoglobulin combined with high-dose aspirin in the acute stage of Kawasaki disease: study protocol for a randomized controlled trial. BMC Pediatr. 2018;18.

18. Huang $X J$, Huang $P$, Zhang $L$, et al. Is aspirin necessary in the acute phase of Kawasaki disease? J Paediatr Child Health. 2018;54:661-4.

19. Platt B, Belarski E, Manaloor J, et al. Comparison of risk of recrudescent fever in children with Kawasaki disease treated with intravenous immunoglobulin and low-dose vs high-dose aspirin. JAMA Netw Open. 2020;3.

20. Zheng XL, Yue P, Liu L, et al. Efficacy between low and high dose aspirin for the initial treatment of Kawasaki disease: current evidence based on a meta-analysis. PLoS One. 2019;14.

21. Migally K, Braunlin EA, Zhang L, Binstadt BA. Duration of high-dose aspirin therapy does not affect long-term coronary artery outcomes in Kawasaki disease. Pediatr Res. 2018:83:1136-45.

22. Pi L, Che D, Long HF, et al. Immature platelets and antiplatelet therapy response to aspirin in Kawasaki disease. Drug Design Development and Therapy. 2018;12:1353-62.

23. Dhanrajani A, Chan M, Pau S, et al. Aspirin dose in Kawasaki disease: the ongoing Battle. Arthritis Care Res. 2018;70:1536-40.

24. Kim GB, Yu JJ, Yoon KL, et al. Medium- or Higher-Dose Acetylsalicylic Acid for Acute Kawasaki Disease and Patient Outcomes. J Pediatr. 2017;184:125-129.e121.

25. Koren G, Silverman E, Sundel R, et al. Decreased protein binding of salicylates in Kawasaki disease. J Pediatr. 1991;118:456-9.

26. Callinan LS, Tabnak F, Holman RC, et al. Kawasaki syndrome and factors associated with coronary artery abnormalities in California. Pediatr Infect Dis J. 2012;31:894-8.

27. Song $\mathrm{D}$, Yeo $\mathrm{Y}, \mathrm{Ha} \mathrm{K}$, et al. Risk factors for Kawasaki disease-associated coronary abnormalities differ depending on age. Eur J Pediatr. 2009;168:1315-21.

\section{Publisher's Note}

Springer Nature remains neutral with regard to jurisdictional claims in published maps and institutional affiliations.

Ready to submit your research? Choose BMC and benefit from:

- fast, convenient online submission

- thorough peer review by experienced researchers in your field

- rapid publication on acceptance

- support for research data, including large and complex data types

- gold Open Access which fosters wider collaboration and increased citations

- maximum visibility for your research: over $100 \mathrm{M}$ website views per year

At $\mathrm{BMC}$, research is always in progress.

Learn more biomedcentral.com/submissions 